\title{
Real-Time Monitoring and Point-of-Care Testing: A Review of the Current Landscape of PrEP Adherence Monitoring
}

This article was published in the following Dove Press journal: Patient Preference and Adherence

\author{
Alisse Hannaford (D) \\ Yotam Arens ${ }^{2}$ \\ Helen Koenig ${ }^{2}$ \\ 'Department of Internal Medicine, \\ Hospital of the University of \\ Pennsylvania, Philadelphia, PA, USA; \\ ${ }^{2}$ Division of Infectious Diseases, Hospital \\ of the University of Pennsylvania, \\ Philadelphia, PA, USA
}

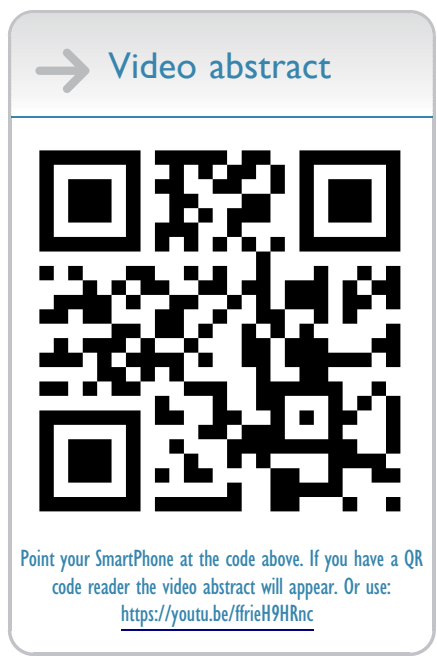

Correspondence: Helen Koenig Division of Infectious Diseases, Hospital of the University of Pennsylvania, MacGregor Infectious Disease Practice, 3400 Civic Center Boulevard, South Pavilion 4th Floor, Philadelphia, PA, 19104, USA

Tel + I 2I5-662-6932

Email helen.koenig@pennmedicine.upenn. edu
Background: Despite pre-exposure prophylaxis (PrEP) being highly effective at preventing HIV, HIV infections among individuals prescribed PrEP continue to occur. The vast majority of these new infections occur among individuals with sub-optimal adherence. One factor that is likely to decrease HIV incidence among PrEP users is a real-time, objective measurement of adherence. Monitoring adherence to PrEP can identify those at risk of becoming lost to follow-up and therefore at greater risk of HIV infection, those in need of additional layers of support to overcome barriers to PrEP, and individuals who need enhanced adherence support. Objective: This paper reviews subjective and objective methods for monitoring PrEP including self-report, drug level monitoring (including serum, plasma, peripheral blood mononuclear cells [PBMC], red blood cell dried blood spots [DBS], hair, and urine) and by measuring participant interaction with the study drug (pill counts, medication event monitoring systems [MEMS] caps).

Clinical Use: A multitude of methods exist for monitoring and supporting adherence. Objective monitoring using DBS and urine will provide a more accurate picture of adherence compared to subjective and non-biomarker objective methods. Preliminary data show that detection of non-adherence using biomarkers, followed by augmented adherence support and counseling, is associated with improved adherence, although more research is needed. PrEP providers will need knowledge of and access to these various strategies, which will require investment and resource allocation from clinics and other PrEP care sites to provide these tools.

Keywords: pre-exposure prophylaxis, PrEP, adherence, adherence monitoring, biomarker

\section{Background}

The United States is home to more than 1.1 million people living with HIV/AIDS and has approximately 35,000 new HIV infections per year, almost $60 \%$ of which are among adolescents and young adults. ${ }^{1}$ There is currently no vaccine that will adequately protect against HIV and there is no cure. Pre-exposure prophylaxis (PrEP) is one of the most important tools to prevent new infections and curb the epidemic. The Centers for Disease Control has estimated that over 1 million individuals are at high risk for HIV acquisition and would benefit from PrEP. ${ }^{2}$ Once-daily oral tenofovir disoproxil fumarate-emtricitabine (TDF-FTC) was the first drug approved by the Federal Drug Administration (FDA) for the prevention of HIV in 2012. Tenofovir alafenamide-emtricitabine (TAF-FTC), was FDA approved for PrEP in 2019 for men who have sex with men and transgender women. ${ }^{3,4}$ Long-acting 
injectable cabotegravir (CAB) administered every 8 weeks is currently undergoing FDA approval.

Despite these advances, new HIV infections among individuals prescribed PrEP continue to occur. While there are a small number of documented seroconversions despite sufficient medication adherence, ${ }^{5-7}$ these are exceptional events, with the vast majority occurring among individuals with sub-optimal adherence and shorter persistence on PrEP. ${ }^{8}$ Having access to PrEP is insufficient to decrease new HIV infections; the prescription must be coupled with counseling and emphasis on adherence. Adherence has been referred to as the "behavioral bridge between PrEP efficacy and effectiveness," in order to realize a widespread reduction in HIV transmission, optimal adherence to PrEP must be achieved. Many studies ${ }^{10-13}$ have drawn the same conclusion when participants take PrEP, it works, and when they do not, it does not.

Indeed, two major original PrEP trials - FEM-PrEP ${ }^{14}$ and VOICE ${ }^{15}$ - failed to show the efficacy of TDF-FTC chemoprophylaxis in the reduction of HIV risk, which was found to be due to poor adherence in these study populations. Conversely, the iPrEx trial found that the risk of HIV acquisition was reduced by more than $90 \%$ with daily oral TDF-FTC. Yet this prophylactic effect was only seen in participants who had detectable drug levels; participants in the intervention arm of the trial with undetectable drug levels had a twelve-fold greater risk of HIV infection than those with detectable drug levels. ${ }^{16}$ Results of HPTN 083 and 084 found that long-acting injectable cabotegravir every 8 weeks was superior to daily oral TDF/FTC for MSM, transgender women and cis-gender women, possibly largely in part due to the injectable medication obviating the need for daily adherence. ${ }^{17,18}$

Why do some people inadequately adhere to PrEP despite presumably striving to avoid an HIV infection? Individuals may experience difficulties with access to PrEP (eg insurance or cost issues, lack of access to healthcare, lack of education about how and where to obtain PrEP), have negative attitudes or beliefs about PrEP (eg PrEP perpetuating the stigma of HIV, perception of promoting condomless sex, inadequate understanding about the science behind PrEP) or may dislike a particular attribute of PrEP (eg perceived to have experienced side effects). ${ }^{19}$ While social network factors often play a role in promoting PrEP, individuals may inadequately adhere if they lack social support (eg lack of support from partner to take PrEP). ${ }^{19}$ Improving adherence requires a multitude of factors, from improving access to PrEP prescriptions, monitoring adherence, identifying barriers and facilitators to adherence, providing feedback to patients regarding adherence patterns, and motivating and encouraging individuals to modify their behaviors to support adequate PrEP adherence.

Real-time, objective measures of adherence for PrEP are essential to assess how and when people are taking medication, and to accelerate a clinical and/or other supportive response for those with inadequate adherence. ${ }^{20,21}$ Quantifying and monitoring adherence to PrEP can identify those at risk of becoming lost to follow-up and therefore at greater risk of HIV infection, ${ }^{22}$ those in need of additional layers of support to overcome barriers to PrEP (impending health insurance loss, harm reduction tools, logistical support such as tokens), and individuals who need enhanced adherence support (more frequent visits, pill boxes, smart pill bottles, etc.). Indeed, metrics of PrEP adherence were strongly associated with future loss to follow-up in a US demonstration project study. ${ }^{22}$ Similarly, we have seen that adherence monitoring for ART with serial viral load testing has been invaluable for chronic ART management. Strategies that enable healthcare workers to measure and understand not only their patients' risk of nonadherence but also their adherence patterns over time might allow them to better allocate (what are often scarce) resources such as counseling, additional staff time, and employment of more sophisticated adherence support tools, to individuals and/or groups of individuals who most need them. While PrEP can either be dosed daily, on-demand, or (in the future) as an injectable given every 4-8 weeks, tools to assess adherence allow clinicians to titrate the amount and type of adherence support resources needed.

Biomarker monitoring for other chronic therapies is in widespread use for tracking adherence. Individuals taking antiretrovirals, lipid-lowering agents or anti-glycemics have markers such as HIV viral load, LDL and Hemoglobin A1C that are checked frequently to help clinicians assess the success or identify red flags in treatment or prevention of the intended disease. These laboratory values are not synonymous with adherence but are used in conjunction with clinical assessment and patient-reported adherence. A full review of technological interventions studied in HIV, including applications for smartphones, is beyond the scope of this paper. Currently, the only routinely monitored adherence marker for individuals on PrEP is an HIV test, and while a positive test result would indicate non-adherence, once an 
individual is positive for HIV, the opportunity to improve adherence is lost. Here we will explore the various tools that have been used to monitor PrEP adherence and how these may impact clinical care and public health.

\section{Methods}

Several procedures were followed to ensure a high-quality review of the literature, including a comprehensive review of PrEP-related manuscripts in peer-reviewed literature, a review of unpublished material presented at national and regional conferences, and a review of the references section of key PrEP manuscripts to identify additional articles to include. Mesh terms used were intentionally kept broad, and were limited to "adherence," "preexposure prophylaxis," "PrEP" and "monitoring." Papers or abstracts were included if they were written in the English language and included original research on, or a review of or commentary on, either objective and/or subjective methods of adherence to PrEP. Four experts in the field of PrEP and/or adherence (1 from within the University of Pennsylvania Health System, and 3 external reviewers) were asked to review the manuscript draft prior to submission and provide constructive criticism, which was subsequently incorporated into this review.

\section{Measuring Adherence}

In the original PrEP trials, investigators quantified adherence using both subjective and objective methods. ${ }^{14,15}$ Self-reported adherence in interviews or surveys is the main subjective tool for quantifying PrEP adherence, which can be monitored over time. Objective assessment of PrEP adherence is characterized by measuring drug concentrations in various body compartments (including serum, plasma, peripheral blood mononuclear cells [PBMC], whole blood cell dried blood spots [DBS], hair, and urine) or by measuring participant interaction with the study drug (pill counts, medication event monitoring systems [MEMS] caps). Notably, this second group of objective measures where the patient interacts with the study drug is manipulatable by the patient. These tools have been summarized in Table 1. As outlined below, objective measurements of adherence have proven to be more strongly associated with HIV risk reduction than subjective assessments.

\section{Self-Reported Adherence}

Self-reported adherence to PrEP is quantified by asking individuals a series of questions about their PrEP use in the last month via in-person interviews or computerized surveys. Multiple studies have shown that self-reported adherence to PrEP does not correlate well with the reduction in risk in HIV acquisition. This was exemplified in the VOICE and FEM-PREP trials. ${ }^{14,15}$ Other trials have shown an inconsistent correlation between self-reported adherence and plasma drug levels. ${ }^{13,23-25}$ Wilson et al validated a three-item measure to ask how many days among the last 30 was a dose missed (0-30), how often the medication was taken as prescribed (never/rarely/ sometimes/usually/almost always/always), and how good a job the patient felt they did at taking the medication as prescribed (very poor/poor/fair/good/very good/ excellent). ${ }^{26}$ Self-reported adherence may not be a reliable tool as it is limited by recall bias, overreporting, and social desirability bias; ${ }^{27,28}$ however, providers may increase reliability by asking the right questions.

\section{Objective Tools for Monitoring PrEP Adherence}

Several objective markers of PrEP adherence have been studied. Tracking pharmacy refills involves using pharmacy records to determine whether or not an individual has picked up their prescription, and if it was filled within the appropriate timeframe. Using these data, a medication possession ratio can be calculated to determine the number of doses of medication that the patient had per time-frame of medication prescribed. ${ }^{29}$ While this method has been shown to be more reliable than self-report in predicting virologic failure among persons living with HIV persons receiving antiretroviral therapy in resource-limited settings, ${ }^{30}$ communication between provider and pharmacy can be labor intensive and does not happen consistently in most practices. To increase efficiency, electronic pharmacy refills that interface with prescribing clinics would streamline data collection, and can even enable clinical alerts to providers when a patient has missed picking up a prescription. $^{31}$ The most significant limitation to monitoring pharmacy refills is the assumption that individuals are taking all doses that they pick up, and this method likely overestimates adherence when compared to other objective measures. $^{32}$

Pill counts have been used either alone or in conjunction with the above methods for monitoring adherence. Patients are given a known number of pills in a medication bottle, and they are instructed to bring this 
Table I Strengths and Weaknesses of Adherence Monitoring Measures

\begin{tabular}{|c|c|c|c|c|}
\hline \multicolumn{2}{|l|}{ Measure } & \multirow{2}{*}{$\begin{array}{l}\text { Strengths } \\
\cdot \text { Inexpensive } \\
\cdot \text { Easy to collect } \\
\cdot \text { Reports of poor adherence likely accurate }\end{array}$} & \multirow{2}{*}{$\begin{array}{l}\text { Weaknesses } \\
\text { - Poorly correlated with adherence } \\
\text { - Limited by recall bias, over-reporting, } \\
\text { and social desirability bias }\end{array}$} & \multirow{2}{*}{$\begin{array}{l}\text { Timeframe } \\
\text { Short term } \\
>\text { long term }\end{array}$} \\
\hline & Self-report & & & \\
\hline & Pharmacy Refills & $\begin{array}{l}\text { Can be used to calculate medication possession } \\
\text { ratio } \\
\text { - Electronic connection between pharmacy and } \\
\text { clinic can alert providers when refills are not } \\
\text { picked up }\end{array}$ & $\begin{array}{l}\text { Can be labor intensive with manual } \\
\text { collection } \\
\text { Overestimates adherence as it assumes } \\
\text { patients take all doses they have picked up }\end{array}$ & $\begin{array}{l}\text { Short and } \\
\text { long term }\end{array}$ \\
\hline & Pill counts & $\begin{array}{l}\cdot \text { Inexpensive } \\
\cdot \text { Easy to collect }\end{array}$ & $\begin{array}{l}\text { Patients must remember to bring pill } \\
\text { bottles to clinic } \\
\text { - Must be compared with medication } \\
\text { possession ratio }\end{array}$ & $\begin{array}{l}\text { Short term } \\
>\text { long term }\end{array}$ \\
\hline & $\begin{array}{l}\text { Electronic } \\
\text { adherence } \\
\text { monitors (EAMs) }\end{array}$ & $\begin{array}{l}\text { Enables day to day monitoring } \\
\text { - Can accurately monitor adherence over time }\end{array}$ & $\begin{array}{l}\text { - Can lose data with technical challenges } \\
\text { - Cost of devices }\end{array}$ & $\begin{array}{l}\text { Short and } \\
\text { long term }\end{array}$ \\
\hline \multirow[t]{5}{*}{$\begin{array}{l}\text { Drug Level } \\
\text { Monitoring }\end{array}$} & Plasma & - Detection correlates with HIV prevention & $\begin{array}{l}\text { - Not commercially available } \\
\text { - Susceptible to "white coat" adherence } \\
\text { - Long turnaround time } \\
\text { - Susceptible to patient pharmacokinetics } \\
\text { - Requires venipuncture }\end{array}$ & $\begin{array}{l}\text { Very short } \\
\text { term }(I-2 \\
\text { days })\end{array}$ \\
\hline & Urine & $\begin{array}{l}\text { - Commercially available } \\
\text { - Detection correlates with HIV prevention } \\
\text { - Rapid turnaround time } \\
\text { - Inexpensive } \\
\text { - Non-invasive and easy to collect } \\
\text { - Highly acceptable to patients }\end{array}$ & $\begin{array}{l}\text { Short-term measure of adherence } \\
\text { Susceptible to "white coat" adherence }\end{array}$ & $\begin{array}{l}\text { Short term } \\
(7-10 \text { days })\end{array}$ \\
\hline & DBS (FTC-TP) & - Well validated & $\begin{array}{l}\text { - Not yet commercially available } \\
\text { - Given not commercially available, } \\
\text { unknown cost to roll out } \\
\text { - Susceptible to patient pharmacokinetics } \\
\text { - Requires fingerstick or venipuncture }\end{array}$ & Short term \\
\hline & $\begin{array}{l}\text { Dried Blood Spot } \\
\text { (DBS) (TFV-DP) }\end{array}$ & $\begin{array}{l}\text { - Commercially available } \\
\text { - Well validated } \\
\text { - Detection correlates with HIV prevention } \\
\text { - Relatively inexpensive } \\
\text { - Adherence over a longer time period }\end{array}$ & $\begin{array}{l}\text { Susceptible to patient pharmacokinetics } \\
\text { Requires fingerstick or venipuncture }\end{array}$ & Long term \\
\hline & Hair & $\begin{array}{l}\text { - Can be stored at room temperature } \\
\text { - Detection correlates with HIV prevention } \\
\text { - Adherence over a longer time period }\end{array}$ & $\begin{array}{l}\text { - Not commercially available } \\
\text { - Mixed data on acceptability given that } \\
150+\text { pieces of hair are needed for each } \\
\text { sample } \\
\text { - Long turnaround time } \\
\text { - Susceptible to patient pharmacokinetics }\end{array}$ & Long term \\
\hline
\end{tabular}

bottle for subsequent visits. In the IPERGAY study, medication was given to patients in 4- and 8-week intervals and a pill count of unused medication was counted at each subsequent visit. ${ }^{12}$ The TDF2 study administered 30 pills at a time. ${ }^{33}$ Pill counts may be labor-intensive during clinic visits as providers need to calculate how many pills a patient should have remaining in their bottles, which varies based on pharmacy refill timing and continuous 
versus on-demand PrEP. Other limitations are that patients may discard their unused medications prior to clinic visits $^{34}$ or forget to bring in their medication bottle, possibly making pill counts no more efficacious than selfreport.

Electronic adherence monitors (EAMs) are devices that enable day-to-day adherence monitoring and have been used for $\mathrm{ART}^{31}$ and tuberculosis treatment ${ }^{35}$ but have yet to be fully explored for PrEP. The most widely known is the Medication Event Monitoring System (MEMS), a medication bottle that records a time and date stamp when the bottle is opened and allows for the transfer of this information to a computer via USB. Day-to -day monitoring provides a pattern of adherence and can enable targeted interventions to decrease missed doses going forward. With traditional MEMs, information is not sent to the provider daily, but rather evaluated over time at scheduled appointments. Newer EAMs such as Wisepill $^{\mathrm{TM}}$ can be linked to phone calls, text messages and/or emails to remind individuals to take their daily dose, or can make a sound once a certain time period has passed without a pill being dispensed as a reminder alarm. Messages can be sent to the prescribed individual, but can also be sent to other individuals (eg friends or providers) who can act as a support group. ${ }^{31}$ This technology, among others, was shown to improve ART adherence in a cohort of rural Ugandans living with HIV. ${ }^{36}$ The automatic reminder strategy may be helpful for daily use of PrEP, although with on-demand use it may be less relevant. Day-to-day dosing patterns can be used to inform counseling sessions; if patients are able to track their periods of heightened HIV risk, this can be compared with the timing of on-demand dosing. Main limitations include technical challenges causing failure of device tracking, possibility for patient manipulation by pocketing pills or opening device without removing a pill, increased staffing requirements, cost of device, requirement for internet access for patients in some cases, and patient convenience. Research is ongoing to evaluate another method: PrEP digital pills. These pills work exactly as typical PrEP pills do with respect to the active medications, but also have a miniature sensor made from silicon, magnesium and copper that emits an electrical pulse when it contacts with stomach acid, sending a Bluetooth signal to an individual's smartphone. These pills would have higher accuracy than EAMs as it would enable individuals to track exactly when and how frequently they are ingesting the medication. ${ }^{37}$
Drug concentrations have been explored as an alternative, objective metric of adherence. An understanding of the pharmacokinetics of oral TDF-FTC, TAF-FTC and $\mathrm{CAB}$ is critical for appreciating the various modalities for drug monitoring. TDF, the prodrug form of TFV, is absorbed in the gut and distributes in the plasma, where it has a short half-life of 2-6 hours. ${ }^{38}$ TFV then enters the intracellular space, where it becomes phosphorylated into the active form, tenofovir diphosphate (TFV-DP), a nucleotide analog with a reverse transcriptase inhibitor effect. TFV-DP concentrates in the intracellular space and has a half-life of 17 days in red blood cells (RBC). TFVDP levels can be measured by dried blood spot, a technique in which $25 \mu \mathrm{l}$ of whole blood is collected via fingerstick or venipuncture. ${ }^{39,40}$ Pharmacokinetics studies in healthy volunteers who receive varying doses of oral TDF-FTC have shown that TFV-DP measurement by DBS reveals average drug exposure over time, giving an estimate of cumulative dosing of the drug. For instance, much like a glycosylated hemoglobin level can estimate glycemic control over a course of 120 days (the life cycle of an average RBC), TFV-DP DBS measurement can estimate how much TDF-FTC an individual has consumed over a period of weeks to months. ${ }^{24}$ DBS was used as the reference standard of objective adherence to PrEP used in the iPrEx trial, where participants with DBS TFV-DP levels of $>700 \mathrm{fmol} /$ punch were found to have a $100 \%$ reduction in risk of HIV acquisition. ${ }^{11}$ Pharmacokinetics studies have shown that TFV-DP levels $\geq 700 \mathrm{fmol} / \mathrm{punch}$ and $\geq 1250 \mathrm{fmol} /$ punch are equivalent to oral TDF-FTC dosing of 4-6 times per week and 7 times per week, respectively. Likewise, TFV-DP in DBS after TAF-FTC dosing was also found to have a long half-life and a linear association with dosing frequency. ${ }^{41}$ In addition, FTC-TP (emtricitabine-triphosphate) has also been studied in DBS for subjects taking TDF-FTC and TAF-FTC; as it has a much shorter half-life, FTC-TP reflects a recent dose much like plasma TFV. ${ }^{40,41}$

Drug level monitoring in hair samples has been explored as a marker for PrEP adherence as well and has been used in HIV seropositive patients to assess ARV adherence. The STRAND study enrolled HIV-negative participants who received oral TDF-FTC either 2, 4, or 7 times per week under directly observed therapy (DOT). ${ }^{42}$ Hair samples were collected by cutting 150-200 strands of hair from the occipital scalp at study visits. ${ }^{43}$ The authors found that TFV levels in hair increased proportionally to the number of doses administered per week. This level can 
Table 2 Interpretation of Urine Tenofovir Adherence Monitoring Results, with Tenofovir (Breakdown Product of Prodrugs TDF and TAF) Adjusted for Urine Creatinine, as Well as the Suggested Clinical Interpretation. Different Day Ranges, ie 7 Days vs 6 Days vs 2 Days are Based on Maximizing Sensitivity and Specificity

\begin{tabular}{|l|l|l|}
\hline $\begin{array}{l}\text { TDF/FTC (Truvada } \\
\text { TMV }\end{array}$ ) \\
Levels & Key & Interpretation \\
\hline$<1: 1$ & $\begin{array}{l}\text { No dose taken in the last } 7 \text { days } \\
\text { No dose taken in the last } 48 \text { hours } \\
\text { Dose taken in the last } 4 \text { days } \\
>22: 1\end{array}$ & $\begin{array}{l}\text { Non adherent in last } 7 \text { days } \\
\text { Non adherent in last } 2 \text { days } \\
\text { Recently adherent in last } 4 \text { days }\end{array}$ \\
\hline \begin{tabular}{l|l|l|} 
TAF/FTC (Descovy ${ }^{\text {TM }}$ ) \\
TFV:Creatinine Control Ratio
\end{tabular} & $\begin{array}{l}\text { No dose taken in the last } 7 \text { days } \\
\text { No dose taken in the last } 48 \text { hours } \\
\text { Dose taken in the last } 6 \text { days }\end{array}$ & $\begin{array}{l}\text { Non adherent in last } 7 \text { days } \\
\text { Non adherent in last } 2 \text { days } \\
\text { Recently adherent in last } 6 \text { days }\end{array}$ \\
\hline $\begin{array}{l}<: 1 \\
>6: 1\end{array}$
\end{tabular}

Abbreviations: TDF/FTC, tenofovir disoproxil fumarate/emtricitabine; TFV, tenofovir; TAF/FTC, tenofovir alafenamide/emtricitabine.

be used to extrapolate the average number of PrEP doses a participant received per week, and whether they are optimally adherent to a protective dose. TFV levels in hair have also been shown to be highly concordant with TFV-DP DBS levels among adolescent PrEP users. ${ }^{25}$ The advantages of using hair samples are that the collection is noninvasive and can be stored at room temperature. Limitations include a prolonged processing time, patient preference, and potential inaccuracies with dyed hair or hair permanent products, thus limiting the generalizability of this tool. Hair sampling has had variably acceptability due to patient hesitation to contribute hair, ${ }^{25}$ and some data suggest lower acceptability with certain hairstyles such as short hair and weaves, which may be more prominent in Black individuals. ${ }^{44,45}$

Plasma measurement of TFV via liquid chromatography with tandem mass spectrometry (LC-MS/MS) has also been studied as an objective marker of PrEP adherence. Plasma TFV levels were measured qualitatively as detectable ( $\geq 10 \mathrm{ng} / \mathrm{mL}$ ) or below the limit of quantitation (BLQ) in multiple studies ${ }^{12-14}$ and may detect dosing of PrEP in the previous $24-48$ hours. ${ }^{38}$ In the PROUD trial, tenofovir was detected in the plasma of all participants who reported adherence. ${ }^{46}$ In the open-label PATH-PrEP study, MSM and transgender women received daily oral TDF-FTC for PrEP and random plasma TFV levels were monitored at study visits. Landovitz et al found that $12 \%$ of participants had undetectable plasma TFV levels during the study period, suggesting no PrEP dosing in the previous 2 days and raising concern for a pattern of suboptimal adherence. Due to its short half-life, random plasma TFV measurement is a short-term measure of adherence and may not accurately predict longer-term PrEP adherence. This may be particularly problematic due to a "white coat" effect of PrEP dosing, where individuals may dose their PrEP prior to a clinic visit to suggest optimal adherence. Limitations of plasma TFV measurement for use in clinical settings are that it is not commercially available and can take up to 3 weeks to process and report.

Urine drug level monitoring is also feasible as TFV is renally excreted into the urine and thus can be detected in urine samples. Urine TFV testing using LCMS/MS has been shown to be highly sensitive for detecting recent adherence to daily TDF-FTC dosing, with a window of detection of at least 7 days. Urine testing was initially validated as a semi-quantitative assay for TDF-based $\operatorname{PrEP}^{47}$ and then was further refined and re-validated on a fully quantitative scale for both TDF-based PrEP and tenofovir alafenamide (TAF)-based PrEP (Table 2) ${ }^{48,49}$ Urine TFV levels can be normalized by using urine creatinine to account for individual variability in TFV metabolism based on creatinine clearance. Figure 1 provides examples of results that are returned to providers.

Urine-based TFV monitoring is particularly appealing due to its noninvasive manner of collection that can be paired with urine-based STI testing, its low cost, and its rapid turn-around time of 2-3 days. Urine testing has been shown to increase adherence to PrEP longitudinally when urine TFV results are provided to patients. ${ }^{50}$ In one study that used urine testing to quantify if patients were non-adherent (no dose in the last 48 


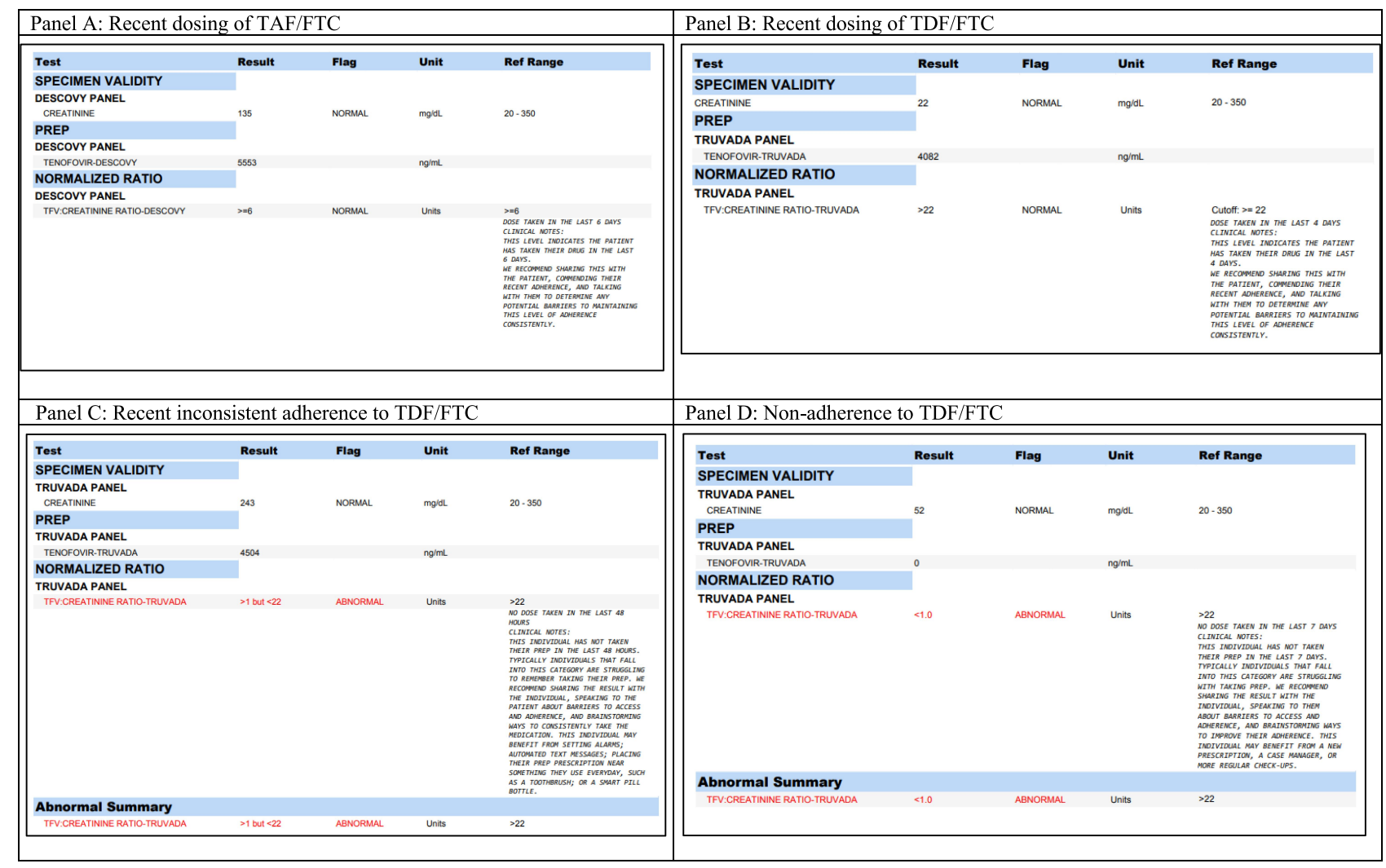

Figure I Examples of laboratory results returned to providers utilizing urine TFV adherence support. Panels A and B are consistent with recent dosing of TAF/FTC and TDF/FTC, respectively. Panel $C$ is consistent with recent inconsistent adherence to TDF/FTC, and Panel D is consistent with nonadherence in the previous 7 days.

hours) versus recently adherent (a dose in the last 6 days), $15 / 17$ (88\%) improved from non-adherence to recently adherent in just one visit. ${ }^{50}$ Non-adherent patients were contacted to discuss barriers to medication use and formulate an adherence plan. ${ }^{51}$ Although a small study, these results suggest that patient adherence can be increased with increased data and targeted counselling. In addition, urine-based TFV monitoring has already been developed into a point-of-care test $\mathrm{t}^{52,53}$ that is currently awaiting FDA approval, where objective data on recent PrEP adherence can be reviewed in real-time with patients to guide further adherence support counseling. In contradistinction to measuring drug concentrations, the TARGET study measured an antibody-based urine TFV assay which was strongly correlated to the LC-MS/MS-based urine assay. $^{54}$ Urine-based adherence monitoring is highly acceptable to younger patients and men of color who have sex with men, as it is noninvasive does not require additional testing outside of routine monitoring PrEP labs; however, "white coat" dosing is a limitation of urine testing. ${ }^{47,55}$ Nevertheless, urine TFV testing is potentially more useful than plasma TFV testing in that the former can distinguish between PrEP dosing in the previous 1-2 days from dosing in the previous 2-7 days. $^{52}$

\section{Clinical Use}

While there is no "gold-standard" for monitoring PrEP adherence, we do have a multitude of methods in our armamentarium for monitoring and supporting adherence. PrEP providers will need knowledge of and access to these various strategies which will require investment and resource allocation from clinics and other PrEP care sites to provide these tools. Furthermore, even as new forms of PrEP delivery such as implantable devices or long-acting injectable medications (cabotegravir) become available and may overcome existing barriers to adherence, oral dosing of PrEP may still be a preferred method of PrEP delivery for many people at high risk of HIV acquisition. Reasons may include short-term use, initial concern for side effects, or concern for the "tail effect" with long-term formulations placing individuals at higher risk of resistance if they do acquire HIV. ${ }^{56}$ In resource-limited settings, uptake of long-acting PrEP will be slow, and TDF- 
FTC will likely continue to be the primary method for PrEP for the next several years.

Two tests that have been commercially available for objective PrEP adherence monitoring are DBS monitoring for TFV-DP ${ }^{57}$ and urine TFV testing. ${ }^{53}$ While DBS monitoring can quantify to some degree the average doses of PrEP per week, it cannot provide insight into variable adherence patterns over time or day-to-day adherence. Urine TFV monitoring cannot quantify TFV dosing over time and is best used to provide data on when the last dose of PrEP was taken within the previous $7-10$ days. Thus, neither is a stand-alone definitive test of adherence, but both can provide clinicians with valuable information to gauge patient adherence over different periods of time. Concentrations of PrEP drug metabolites are lower in the female genital tract compared with the rectum. ${ }^{14,15}$ Being aware of patient gender and type of sexual activity that a patient is engaging in is necessary as the adherence threshold differs by the site of exposure. Tests for TFV-DP levels will have different cut-offs which can be extrapolated to confer "rectally protective dosing" and "vaginally protective dosing." Urine tests correlate with recent adherence and can differentiate between non-adherence and inconsistent adherence over the last week (Table 2). Examples of laboratory results returned to providers utilizing urine TFV adherence support can be seen in Figure 1, with examples of recent dosing, recent inconsistent dosing and non-adherence.

Notably, all of the objective methods are more informative than self-reported adherence alone, with the caveat that self-reported non-adherence correlates well with nonadherence. ${ }^{58}$ Using a combined approach to adherence monitoring, with the increased roll-out of commercial methods to monitor plasma, DBS and urine drug levels in addition to expanding and electronically facilitating monitoring of pharmacy refills and EAM devices would provide a toolbox of options for providers to choose from. It is important to note that urine and plasma TFV measurement can detect short-term PrEP adherence over the course of days, while DBS TFV-DP and hair TFV measurement are better suited to detect long-term adherence to PrEP, which can then be extrapolated to average dosing per week. However, average dosing per week is limited in how well it predicts day-to-day adherence. As data from each of these objective markers offer patients and their providers a picture of adherence over a different window of time, they may be used in conjunction to obtain a more complete picture of adherence, similar to using a glucometer for daily blood glucose monitoring as well as a glycosylated hemoglobin every 3 months in diabetic patients. Individuals will continue to acquire HIV even while prescribed PrEP if we do not increase our ability to monitor adherence. With these tools and the information gleaned from them, healthcare workers can curate the volume of resources given to a particular individual, providing targeted counseling and increased resource allocation to those who need it the most.

A biopsychosocial framework will also help providers navigate monitoring adherence in the setting of preventioneffective adherence, a term coined by Haberer et al to take into account dynamic HIV acquisition risk behaviors. ${ }^{59}$ As an individual's risk for HIV acquisition changes over time, there will be periods of time when an individual is at considerably lower risk for HIV (eg in a long-term monogamous relationship or during the COVID-19 pandemic). High PrEP adherence is important during periods of risk, as adherence in the absence of risk confers cost, and potential side effects and toxicity without benefit. ${ }^{32}$ It can be difficult to predict when an individual will be at high risk; thus, the need for "seasons of protection" that are conservative estimates of risk. ${ }^{60}$ Helping individuals understand specifically when they should be taking PrEP may alleviate some of the barriers to adherence. Conversations between providers and patients about risk over time will help determine whether daily oral PrEP is the best tool for a given individual, or whether another dosing strategy would be more appropriate (eventdriven PrEP, post-exposure prophylaxis for individuals with infrequent sexual encounters or long-acting injectables) with the hope that once an individual is on the best method for their lifestyle, it will be easier to achieve optimal adherence. Additionally, adherence can be influenced by implicit judgements; frameworks have been developed to further understand patients' perspectives on medication adherence taking into account their personal beliefs and concerns. While not yet validated for PrEP, these frameworks may serve as an additional tool for improving adherence outcomes. ${ }^{61}$ Pairing adherence monitoring with specific interventions to target these drivers of suboptimal adherence will be paramount.

\section{Further Directions}

A key gap in our current landscape of adherence is data to demonstrate that detection of suboptimal adherence leads to improvements in adherence and ultimately lower the risk of HIV infection. While Landovitz et al showed that a modest improvement in long-term adherence was achieved when suboptimal TFV detection in plasma was coupled with intensive adherence counseling term, ${ }^{13}$ the 
scalability and sustainability of this intervention are unclear. Preliminary data show that detection of PrEP nonadherence by urine TFV monitoring, followed by augmented adherence support counseling, was associated with increased urine TFV detection, and thus PrEP adherence, at subsequent clinic visits. ${ }^{50}$

Furthermore, varying levels of adherence may be sufficient to prevent HIV infection in different populations, as we know that protective drug levels vary by risk group. Although overall PrEP uptake is increasing, certain populations in the United States continue to be at increased risk of HIV infection with disparities persisting among minority groups. In particular, adolescent and young adults, as well as black and Latino MSM, are at the highest risk. ${ }^{62}$ Social and cultural contexts will be important to consider when designing adherence monitoring strategies. Addressing these gaps in care and finding effective ways to help patients improve their adherence in a populationspecific manner will be imperative to reducing the overall incidence and burden of HIV.

\section{Disclosure}

Dr Helen Koenig reports personal fees from UrSure Inc and ViralEd; grants from Gilead, outside the submitted work. The authors report no other conflicts of interest in this work.

\section{References}

1. Centers for Disease Control and Prevention. HIV in the United States and Dependent Areas. CDC; 2020.

2. Centers for Disease Control and Prevention. HIV prevention pill not reaching most Americans who could benefit - especially people of color. 2018.

3. US Food and Drug Administration. FDA approves second drug to prevent HIV infection as part of ongoing efforts to end the HIV epidemic. 2019

4. IDSE Infectious Disease Special Edition. FDA Advisory Committee Endorses Descovy for PrEP for MSM. 2019.

5. Knox DC, Anderson PL, Harrigan PR, Tan DH. Multidrug-resistant HIV-1 infection despite preexposure prophylaxis. $N$ Engl $\mathrm{J} \mathrm{Med}$. 2017;376:501-502. doi:10.1056/NEJMc1611639

6. Markowitz M, Grossman H, Anderson PL, et al. Newly acquired infection with multidrug-resistant HIV-1 in a patient adherent to preexposure prophylaxis. J Acquir Immune Defic Syndr. 2017;76:e104e6. doi:10.1097/QAI.0000000000001534

7. Hoornenborg E, Prins M, Achterbergh RCA, et al. Acquisition of wild-type HIV-1 infection in a patient on pre-exposure prophylaxis with high intracellular concentrations of tenofovir diphosphate: a case report. Lancet HIV. 2017;4:e522-e8. doi:10.1016/S2352-3018(17) 30132-7

8. Eaton LA, Matthews DD, Bukowski LA, et al. Elevated HIV prevalence and correlates of PrEP use among a community sample of black men who have sex with men. J Acquir Immune Defic Syndr. 2018;79:339-346. doi:10.1097/QAI.0000000000001822
9. Amico KR. Adherence to preexposure chemoprophylaxis: the behavioral bridge from efficacy to effectiveness. Curr Opin HIV AIDS. 2012;7:542-548. doi:10.1097/COH.0b013e3283582d4a

10. van der Straten A, Van Damme L, Haberer JE, Bangsberg DR. Unraveling the divergent results of pre-exposure prophylaxis trials for HIV prevention. AIDS (London, England). 2012;26:F13-F19. doi:10.1097/QAD.0b013e3283522272

11. Grant RM, Anderson PL, McMahan V, et al. Uptake of pre-exposure prophylaxis, sexual practices, and HIV incidence in men and transgender women who have sex with men: a cohort study. Lancet Infect Dis. 2014;14:820-829. doi:10.1016/S14733099(14)70847-3

12. Molina JM, Capitant C, Spire B, et al. On-demand preexposure prophylaxis in men at high risk for HIV-1 Infection. $N$ Engl J Med. 2015;373:2237-2246. doi:10.1056/NEJMoa1506273

13. Landovitz RJ, Beymer M, Kofron R, et al. Plasma tenofovir levels to support adherence to $\mathrm{TDF} / \mathrm{FTC}$ preexposure prophylaxis for HIV prevention in MSM in Los Angeles, California. J Acquir Immune Defic Syndr. 2017;76:501-511. doi:10.1097/QAI.000000 0000001538

14. Van Damme L, Corneli A, Ahmed K, et al. Preexposure prophylaxis for HIV infection among African women. $N$ Engl $J$ Med. 2012;367:411-422. doi:10.1056/NEJMoa1202614

15. Marrazzo JM, Ramjee G, Richardson BA, et al. Tenofovir-based preexposure prophylaxis for HIV infection among African women. N Engl J Med. 2015;372:509-518. doi:10.1056/NEJMoa1402269

16. Grant RM, Lama JR, Anderson PL, et al. Preexposure chemoprophylaxis for HIV prevention in men who have sex with men. $N$ Engl $J$ Med. 2010;363:2587-2599. doi:10.1056/NEJMoa1011205

17. HPTN Trial Network. HPTN 083 Study Summary. 2020.

18. HPTN Trial Network. HPTN 084 Study Summary. 2020.

19. Hannaford A, Lipshie-Williams M, Starrels JL, et al. The use of online posts to identify barriers to and facilitators of HIV Pre-exposure Prophylaxis (PrEP) among men who have sex with men: a comparison to a systematic review of the peer-reviewed literature. AIDS Behav. 2018;22:1080-1095. doi:10.1007/s10461017-2011-3

20. Drain PK, Bardon AR, Simoni JM, et al. Point-of-care and near real-time testing for antiretroviral adherence monitoring to HIV treatment and prevention. Curr HIV/AIDS Rep. 2020;17:487-498. doi:10.1007/s11904-020-00512-3

21. Bardon AR, Simoni JM, Layman LM, Stekler JD, Drain PK. Perspectives on the utility and interest in a point-of-care urine tenofovir test for adherence to HIV pre-exposure prophylaxis and antiretroviral therapy: an exploratory qualitative assessment among U.S. clients and providers. AIDS Res Ther. 2020;17:50. doi:10.1186/s12981-020-00308-w

22. Spinelli MA, Glidden DV, Anderson PL, et al. Brief report: short-term adherence marker to PrEP predicts future nonretention in a large PrEP demo project: implications for point-of-care adherence testing. $J$ Acquir Immune Defic Syndr. 2019;81:158-162. doi:10.1097/QAI.0000000000002005

23. Abdool Karim Q, Abdool Karim SS, Frohlich JA, et al. Effectiveness and safety of tenofovir gel, an antiretroviral microbicide, for the prevention of HIV infection in women. Science (New York, NY). 2010;329:1168-1174.

24. Baxi SM, Liu A, Bacchetti P, et al. Comparing the novel method of assessing PrEP adherence/exposure using hair samples to other pharmacologic and traditional measures. J Acquir Immune Defic Syndr. 2015;68:13-20. doi:10.1097/QAI.0000000000000386

25. Koss CA, Hosek SG, Bacchetti P, et al. Comparison of measures of adherence to human immunodeficiency virus preexposure prophylaxis among adolescent and young men who have sex with men in the United States. Clin Infect Dis. 2018;66:213-219. doi:10.1093/cid/ $\operatorname{cix} 755$ 
26. Wilson IB, Lee Y, Michaud J, Fowler FJ, Rogers WH. Validation of a new three-item self-report measure for medication adherence. AIDS Behav. 2016;20:2700-2708. doi:10.1007/s10461-016-1406-x

27. Hendrix CW, Andrade A, Bumpus NN, et al. Dose frequency ranging pharmacokinetic study of tenofovir-emtricitabine after directly observed dosing in healthy volunteers to establish adherence benchmarks (HPTN 066). AIDS Res Hum Retroviruses. 2016;32:32-43. doi:10.1089/aid.2015.0182

28. Krumpal I. Determinants of social desirability bias in sensitive surveys: a literature review. Qual Quant. 2013;47:2025-2047. doi:10.1007/s11135-011-9640-9

29. Messou E, Kouakou M, Gabillard D, et al. Medication possession ratio: predicting and decreasing loss to follow-up in antiretroviral treatment programs in Côte d'Ivoire. J Acquir Immune Defic Syndr. 2011;57(Suppl 1):S34-S39. doi:10.1097/QAI.0b013e3182208003

30. Sangeda RZ, Mosha F, Prosperi M, et al. Pharmacy refill adherence outperforms self-reported methods in predicting HIV therapy outcome in resource-limited settings. BMC Public Health. 2014;14:1035. doi:10.1186/1471-2458-14-1035

31. Bell KM, Haberer JE. Actionable adherence monitoring: technological methods to monitor and support adherence to antiretroviral therapy. Curr HIV/AIDS Rep. 2018;15:388-396. doi:10.1007/ s11904-018-0413-0

32. Haberer JE, Bangsberg DR, Baeten JM, et al. Defining success with HIV pre-exposure prophylaxis: a prevention-effective adherence paradigm. AIDS (London, England). 2015;29:1277-1285. doi:10.1097/QAD.0000000000000647

33. Thigpen MC, Kebaabetswe PM, Paxton LA, et al. Antiretroviral preexposure prophylaxis for heterosexual HIV transmission in Botswana. $N$ Engl J Med. 2012;367:423-434. doi:10.1056/ NEJMoa1110711

34. Okatch H, Beiter K, Eby J, et al. Brief report: apparent antiretroviral overadherence by pill count is associated with HIV treatment failure in adolescents. J Acquir Immune Defic Syndr. 2016;72:542-545. doi:10.1097/QAI.0000000000000994

35. Liu X, Lewis JJ, Zhang H, et al. Effectiveness of electronic reminders to improve medication adherence in tuberculosis patients: a cluster-randomised trial. PLoS Med. 2015;12:e1001876. doi:10.1371/journal.pmed.1001876

36. Haberer JE, Musiimenta A, Atukunda EC, et al. Short message service (SMS) reminders and real-time adherence monitoring improve antiretroviral therapy adherence in rural Uganda. AIDS (London, England). 2016;30:1295-1300. doi:10.1097/ QAD.0000000000001021

37. Abbasi J. HIV preexposure prophylaxis "smart pill" in early testing. JAMA. 2018;320:965.

38. Hawkins T, Veikley W, St Claire RL, Guyer B, Clark N, Kearney BP. Intracellular pharmacokinetics of tenofovir diphosphate, carbovir triphosphate, and lamivudine triphosphate in patients receiving triple-nucleoside regimens. $J$ Acquir Immune Defic Syndr. 2005;39:406-411. doi:10.1097/01.qai.0000167155.44980.e8

39. Castillo-Mancilla JR, Zheng JH, Rower JE, et al. Tenofovir, emtricitabine, and tenofovir diphosphate in dried blood spots for determining recent and cumulative drug exposure. AIDS Res Hum Retroviruses. 2013;29:384-390. doi:10.1089/aid.2012.0089

40. Anderson PL, Liu AY, Castillo-Mancilla JR, et al. Intracellular tenofovir-diphosphate and emtricitabine-triphosphate in dried blood spots following directly observed therapy. Antimicrob Agents Chemother. 2018;62.

41. Yager J, Castillo-Mancilla J, Ibrahim ME, et al. Intracellular tenofovir-diphosphate and emtricitabine-triphosphate in dried blood spots following tenofovir alafenamide: the TAF-DBS study. $J$ Acquir Immune Defic Syndr. 2020;84:323-330. doi:10.1097/ QAI.0000000000002354
42. Saberi P, Ming K, Legnitto D, Neilands TB, Gandhi M, Johnson MO. Feasibility and acceptability of novel methods to estimate antiretroviral adherence: a longitudinal study. PLoS One. 2019;14:e210791. doi:10.1371/journal.pone.0210791

43. Liu AY, Yang Q, Huang Y, et al. Strong relationship between oral dose and tenofovir hair levels in a randomized trial: hair as a potential adherence measure for pre-exposure prophylaxis (PrEP). PLoS One. 2014;9:e83736. doi:10.1371/journal.pone.0083736

44. Hickey MD, Salmen CR, Tessler RA, et al. Antiretroviral concentrations in small hair samples as a feasible marker of adherence in rural Kenya. J Acquir Immune Defic Syndr. 2014;66:311-315. doi:10.1097/ QAI.0000000000000154

45. Gandhi M, Murnane PM, Bacchetti P, et al. Hair levels of preexposure prophylaxis drugs measure adherence and are associated with renal decline among men/transwomen. AIDS (London, England). 2017;31:2245-2251. doi:10.1097/QAD.0000000000001615

46. McCormack S, Dunn DT, Desai M, et al. Pre-exposure prophylaxis to prevent the acquisition of HIV-1 infection (PROUD): effectiveness results from the pilot phase of a pragmatic open-label randomised trial. Lancet (London, England). 2016;387:53-60. doi:10.1016/ S0140-6736(15)00056-2

47. Koenig HC, Mounzer K, Daughtridge GW, et al. Urine assay for tenofovir to monitor adherence in real time to tenofovir disoproxil fumarate/emtricitabine as pre-exposure prophylaxis. HIV Med. 2017;18:412-418. doi:10.1111/hiv.12518

48. Moorthy GS, Lalley-Chareczko L, Koenig HC, Zuppa AF. Tenofovir urine assay to monitor adherence to HIV pre-exposure prophylaxis (PrEP). Curr Clin Pharmacol. 2019.

49. Lalley-Chareczko L, Hiserodt E, Moorthy G, Zuppa A, Mounzer K, Koenig $\mathrm{H}$. Urine assay to measure tenofovir concentrations in patients taking tenofovir alafenamide. Front Pharmacol. 2020;11:286. doi:10.3389/fphar.2020.00286

50. Daughtridge G, Malone-Thomas S Use of a Urine Adherence Test Coupled with Adherence Intervention to Increase PrEP Adherence. Fast-Track Cities London. 2019.

51. Daughtridge G, Hebel S, Hashim J, et al. Urine assay for tenofovir to monitor real-time adherence to tenofovir disoproxil fumarate and tenorovir alafenamide. HIV/AIDS J. 2020.

52. Gandhi M, Wang G, King R, et al. Development and validation of the first point-of-care assay to objectively monitor adherence to HIV treatment and prevention in real-time in routine settings. AIDS (London, England). 2020;34:255-260. doi:10.1097/QAD.0000000000002395

53. Patient-friendly diagnostic tests that measure and improve adherence to medications; 2020. Available from: https://www.ursureinc.com/. Accessed January 21, 2021

54. Drain PK, Kubiak RW, Siriprakaisil O, et al. Urine tenofovir concentrations correlate with plasma and relate to tenofovir disoproxil fumarate adherence: a randomized, directly observed pharmacokinetic trial (TARGET study). Clin Infect Dis. 2020;70:2143-2151. doi:10.1093/cid/ciz645

55. Kowalczyk Mullins TL, Braverman PK, Dorn LD, Kollar LM, Kahn JA. Adolescent preferences for human immunodeficiency virus testing methods and impact of rapid tests on receipt of results. J Adolesc Health. 2010;46:162-168. doi:10.1016/j. jadohealth.2009.06.015

56. Landovitz R. Tail-Phase Safety, Tolerability and Pharmacokinetics of Long-Acting Injectable Cabotegravir in HIV-Uninfected Individuals: HPTN 077 Final Results. Madrid: HIV Research for Prevention conference (HIVR4P 2018); 2018.

57. Diagnostics Delivered; 2020. Available from: https://moleculartestin glabs.com/. Accessed January 21, 2021.

58. Amico KR, Marcus JL, McMahan V, et al. Study product adherence measurement in the iPrEx placebo-controlled trial: concordance with drug detection. J Acquir Immune Defic Syndr. 2014;66:530-537. doi:10.1097/QAI.0000000000000216 
59. Amico KR, Mansoor LE, Corneli A, Torjesen K, van der Straten A. Adherence support approaches in biomedical HIV prevention trials: experiences, insights and future directions from four multisite prevention trials. AIDS Behav. 2013;17:2143-2155. doi:10.1007/s10461013-0429-9

60. Haberer JE. Current concepts for PrEP adherence in the PrEP revolution: from clinical trials to routine practice. Curr Opin HIV AIDS. 2016;11:10-17. doi:10.1097/COH.000000000000220
61. Horne R, Chapman SC, Parham R, Freemantle N, Forbes A, Cooper V. Understanding patients' adherence-related beliefs about medicines prescribed for long-term conditions: a meta-analytic review of the Necessity-Concerns Framework. PLoS One. 2013;8: e80633.

62. CDC. Diagnoses of HIV infection in the United States and dependent areas. 2018-2019.

\section{Publish your work in this journal}

Patient Preference and Adherence is an international, peer-reviewed, open access journal that focusing on the growing importance of patient preference and adherence throughout the therapeutic continuum. Patient satisfaction, acceptability, quality of life, compliance, persistence and their role in developing new therapeutic modalities and compounds to optimize clinical outcomes for existing disease states are major areas of interest for the journal. This journal has been accepted for indexing on PubMed Central. The manuscript management system is completely online and includes a very quick and fair peer-review system, which is all easy to use. Visit http:// www.dovepress.com/testimonials.php to read real quotes from published authors. 